\title{
Classification of primary and incisional abdominal wall hernias
}

\author{
F. E. Muysoms - M. Miserez - F. Berrevoet - G. Campanelli - G. G. Champault - E. Chelala \\ U. A. Dietz $\cdot$ H. H. Eker - I. El Nakadi - P. Hauters $\cdot$ M. Hidalgo Pascual · A. Hoeferlin · \\ U. Klinge $\cdot$ A. Montgomery $\cdot$ R. K. J. Simmermacher $\cdot$ M. P. Simons $\cdot$ M. Śmietański • \\ C. Sommeling $\cdot$ T. Tollens $\cdot$ T. Vierendeels $\cdot$ A. Kingsnorth
}

Received: 9 February 2009/ Accepted: 7 May 2009/Published online: 3 June 2009

(c) The Author(s) 2009. This article is published with open access at Springerlink.com

\begin{abstract}
Purpose A classification for primary and incisional abdominal wall hernias is needed to allow comparison of publications and future studies on these hernias. It is important to know whether the populations described in different studies are comparable.

Methods Several members of the EHS board and some invitees gathered for 2 days to discuss the development of
\end{abstract}

Results of a consensus meeting on the development of an EHS classification held in Ghent, Belgium, 2-4 October 2008.

\section{F. E. Muysoms ( $\square)$}

Department of Surgery, AZ Maria Middelares,

Kortrijksesteenweg 1026, 9000 Ghent, Belgium

e-mail: filip.muysoms@azmmsj.be

\section{Miserez}

Department of Abdominal Surgery,

Universitair Ziekenhuis Leuven, Herestraat 49,

3000 Leuven, Belgium

F. Berrevoet

Department of General and Hepatobiliary Surgery,

University Hospital Ghent, 2K12 IC De Pintelaan 185,

9000 Ghent, Belgium

\section{G. Campanelli}

Department of Surgical Sciences, Multimedica Hospital,

University of Insubria-Varese, Castellanza, Italy

G. G. Champault

Chef du service de chirurgie,

Centre Hospitalo-universitaire Jean Verdier digestive,

Université Paris XIII, Avenue du 14 Juillet,

93140 Bondy, France

E. Chelala

Department of Digestive and Endocrine Surgery,

Centre Hospitalier Universitaire de Tivoli,

Avenue Max Buset 34, 7100 La Louvière, Belgium an EHS classification for primary and incisional abdominal wall hernias.

Results To distinguish primary and incisional abdominal wall hernias, a separate classification based on localisation and size as the major risk factors was proposed. Further data are needed to define the optimal size variable for classification of incisional hernias in order to distinguish subgroups with differences in outcome.

Conclusions A classification for primary abdominal wall hernias and a division into subgroups for incisional

U. A. Dietz

Klinik und Poliklinik für Allgemein-, Viszeral-,

Gefäß- und Kinderchirurgie, Universitätsklinikum Würzburg,

Oberdürrbacher Strasse 6, 97080 Würzburg, Germany

\section{H. H. Eker}

Department of Surgery, Erasmus Medical Center,

's Gravendijkwal 230, 3051, CE, Rotterdam, The Netherlands

I. El Nakadi

Clinic of Abdominal Wall and Upper GI Surgery,

Erasme Hospital, 808 Route de Lennik, 1070 Brussels, Belgium

\section{P. Hauters}

Department of Surgery, Clinique Notre-Dame,

Avenue Delmee 2, 7500 Tournai, Belgium

M. Hidalgo Pascual

Department of General and Digestive Surgery,

Hospital Universitario 12 de Octubre, Madrid, Spain
A. Hoeferlin
Hernienpraxis Mainz, Rheinstr. 4 Fort Malakoff,
55116 Mainz, Germany
U. Klinge
Department of Applied Medical Engineering,
Helmholtz Institute, RWTH Aachen University,
Pauwelsstraße 20, 52074 Aachen, Germany 
abdominal wall hernias, concerning the localisation of the hernia, was formulated.

Keywords Abdominal wall hernia - Classification . Incisional hernia - Ventral hernia - Umbilical hernia . Epigastric hernia

\section{Introduction}

At the 29th Congress of the European Hernia Society in Athens in May 2007, Andrew Kingsnorth, the president of the EHS, stressed that a classification of ventral and incisional hernias is important because at this moment we are comparing "apples and oranges" in the different studies that are published and presented at meetings [1].

Already in 2000, Schumpelick stated that a classification of incisional hernias, like we have for groin hernias, is urgently needed. "Despite the magnitude of the problem, we do not have a classification that is simple, reproducible and internationally accepted" [2].

Since 2000, several authors have proposed classifications for incisional hernias, but none of them are widely used in the literature on incisional hernias [2-5].

\author{
A. Montgomery \\ Department of Surgery, Malmö University Hospital, \\ 20502 Malmö, Sweden
}

R. K. J. Simmermacher

Department of Surgery, University Hospital, 3584, CX, Utrecht, The Netherlands

\section{P. Simons}

Department of Surgery, Onze Lieve Vrouwe Gasthuis, Oosterpark 9, 1091, AC, Amsterdam, The Netherlands

\section{M. Śmietański}

Department of General and Endocrine Surgery and Transplantation, Medical University of Gdańsk,

7 Debinki Street, 80-211 Gdańsk, Poland

\section{Sommeling}

Department of Surgery, O.L.V. van Lourdes Ziekehuis, Vijfseweg 150, 8790 Waregem, Belgium

\section{T. Tollens}

Department of Surgery, Imelda ziekenhuis, Imeldalaan 9, 2820 Bonheiden, Belgium

\section{T. Vierendeels}

Department of Abdominal Surgery, ASZ Campus Aalst, Merestraat 80, 9300 Aalst, Belgium

\author{
A. Kingsnorth \\ Peninsula Medical School, Derriford Hospital, Level 7, \\ Plymouth PL6 8DH, UK
}

\section{Materials and methods}

\section{Methodology}

Several members of the EHS board and some invitees gathered at the initiative of the Belgian Section for Abdominal Wall Surgery (BSAWS) and the Dutch Hernia Society (DHS) for 2 days to discuss the development of an EHS classification for primary and incisional abdominal wall hernias. ${ }^{1}$

During an initial discussion, the existing proposals were briefly presented by one of the participants.

Thereafter, a decision was taken concerning the purpose of a classification and the scope of this consensus meeting. Some of the participants saw it mainly as a search for a simple classification. Because it was supported by and originated from the EHS, this classification could have a greater application in hospitals and in the surgical literature than the previous proposals published originating from one centre. Others were more in favour of an open structured approach, in which "scientists" would gather a maximum number of data sets in a prospective registry. With this registry, it was hoped to discover the most valuable and important risks factors for recurrence in order to direct future guidelines and therapeutic choices. It was decided to focus first on a simple, reproducible classification, because getting results out of the registry may take many years. A classification was proposed as such, including localisation of the hernia and the size of the hernia defect as decisive for the outcome, not going into its use to direct therapeutic choices for the present time. During the last session of the

\footnotetext{
${ }^{1}$ At the initiative of the first author, Filip Muysoms, current president of the Belgian Section for Abdominal Wall Surgery (BSAWS), and in collaboration with Rogier Simmermacher [member of the Dutch Hernia Society (DHS) and Secretary for Educational of the European Hernia Society (EHS)] and with Marc Miserez (member of BSAWS and Secretary Scientific Research of the EHS), a consensus meeting on the classification of primary and incisional abdominal wall hernias was organised. The BSAWS and the DHS are the National Chapters of the EHS, respectively from Belgium and The Netherlands. A first preparatory meeting took place with members of both Chapters during a whole day session in La Hulpe, Belgium, on 4 April 2008. This was followed by a second meeting in Brussels, Belgium, on 16 September 2008.

As participants to the consensus meeting, held in Ghent, Belgium, on 2-4 October 2008, we invited the board members and past presidents of the EHS (A. Kingsnorth, G. Campanelli, G.G. Champault, A. Hoeferlin, S. Mandala, M. Miserez, R.K.J. Simmermacher, M. Śmietański, J.B. Flament and M. Hidalgo), the board members of the BSAWS (F.E. Muysoms, F. Berrevoet, E. Chelala, I. El Nakadi, P. Hauters, C. Sommeling, T. Tollens and T. Vierendeels) and the board members of the DHS (H.H. Eker and M.P. Simons). In addition we invited some other European experts (U.A. Dietz, U. Klinge and A. Montgomery) who by publications and organisation of national registries have shown major interest in hernia classification.
} 
meeting, the development of a large, broad and open structured European registry was initiated.

\section{Currently existing classifications}

Chevrel and Rath [3] proposed a classification for incisional hernias in 2000. This classification is attractive, because it is simple, and the data required to reach the classification are readily obtained. Three parameters were utilised. Firstly, the localisation of the hernia of the abdominal wall: divided into median (M1-M4) and lateral (L1-L4) hernias. Secondly, the size of the hernia: it was postulated that the width of the hernia defect is the most important parameter (greater than hernia defect surface, length of the hernia or size of the hernia sac), which was divided into four groups (W1-W4). As a third parameter of this classification, subgroups were made for incisional hernias and recurrences: the number of previous hernia repairs was recorded as $(\mathrm{R} 0, \mathrm{R} 1, \mathrm{R} 2, \mathrm{R} 3, \ldots)$. Although apparently easy to use, this classification has not been commonly used in the literature.

In his book on hernia surgery, "Hernien", Schumpelick described a classification that divided incisional hernias into five classes [2]. The size of the defect, the clinical aspect of the hernia in lying and standing position, the localisation of the incision and the number of previous repairs were used for this classification.

Korenkov et al. [4] reported on the results of an expert meeting on classification and surgical treatment of incisional hernia, but no detailed classification proposal resulted from this meeting.

Ammaturo and Bassi [6] suggested an additional parameter to the Chevrel classification. The ratio between the anterior abdominal wall surface and the wall defect surface predicts a strong abdominal wall tension when closing the defect, with possible abdominal compartment syndrome development, and thus might influence the choice of surgical technique.

Recently, Dietz et al. [5] proposed another alternative classification of incisional hernias in which variables like body type, hernia morphology and risk factors for recurrence were included and recommendations made for surgical repair based on the different types. It is based on a self-explanatory taxonomy and is intended to tailor the repair to the body type and risk factors of the individual patient.

The Swedish Abdominal Wall Hernia Registry presented their data collection sheet for incisional and ventral hernias at the EAES congress in Stockholm in June 2008, which forms the basis for a classification and includes many prognostic relevant variables. For this reason Agneta Montgomery was invited to the consensus meeting to present the method of classification used in Sweden.

\section{Purpose of a classification}

The primary purpose of any classification should be to improve the possibility of comparing different studies and their results. By describing hernias in a standardised way, different patient populations can be compared. The secondary purpose of a classification would be to collect results of different surgical techniques from the literature and develop evidence-based therapeutic guidelines using the classification. When a classification would become generally accepted, future studies might use the subgroups within the classification in their prospective registries and within the inclusion criteria for prospective studies.

Scope of the classification: primary ventral hernias versus incisional ventral hernias

The first decision to take was whether the classification would involve primary ventral hernias and incisional ventral hernias in one classification or if two separate classifications were preferable. A consensus was reached on the decision to separate the two entities, since in the authors' opinion primary ventral hernias have a different aetiopathology compared with incisional abdominal wall hernias resulting from failure of a previous incision. The group reached agreement on separating non-incisional hernias, "primary abdominal wall hernias" (also known as "ventral"). and the other "incisional abdominal wall hernias". A recurrent hernia after a primary abdominal wall hernia treatment will then fall into the incisional hernia group. To avoid confusion, the word "primary incisional hernia" should not be used.

There was a consensus to exclude "parastomal hernias" from this classification. Although they are by definition incisional hernias, they make up a distinct group, with specific properties and treatment options.

Format of the classification

In 2007 the EHS published a simple classification for groin hernias [7]. We agreed that a classification for primary abdominal wall hernias and incisional hernias should preferably be in a similar format to the EHS groin hernia classification. This would involve the development of a grid format for the classification, although this may place restrictions on the number of variables that can be used in this classification.

Variables for classification

When proposing a classification, it is important to determine the most suitable variables to include in the classification. However, it is important to keep a classification 
Table 1 Possible variables to use for classifying primary and incisional abdominal wall hernias and their use in previous classifications

\begin{tabular}{|c|c|c|c|c|c|c|}
\hline $\begin{array}{l}\text { Variables for classification } \\
\text { of primary or incisional } \\
\text { abdominal wall hernias }\end{array}$ & $\begin{array}{l}\text { Chevrel } \\
\text { and Rath [3] }\end{array}$ & $\begin{array}{l}\text { Korenkov } \\
\text { et al. [4] }\end{array}$ & Schumpelick[2] & $\begin{array}{l}\text { Ammaturo } \\
\text { and Bassi [6] }\end{array}$ & $\begin{array}{l}\text { Swedish } \\
\text { registry }\end{array}$ & $\begin{array}{l}\text { Dietz et al. } \\
{[5]}\end{array}$ \\
\hline $\begin{array}{l}\text { Size of the hernia defect: surface area, } \\
\text { length, width }\end{array}$ & Width & Width or length & Maximal size & Width & Width and length & Width and length \\
\hline \multicolumn{7}{|l|}{ Size of the hernia sac } \\
\hline Number of hernia defects & & & $\times$ & & $\times$ & \\
\hline BMI of the patient & & & & & $\times$ & $\times$ \\
\hline $\begin{array}{l}\text { Ratio anterior abdominal wall surface/ } \\
\text { wall defect surface }\end{array}$ & & & & $\times$ & & \\
\hline \multicolumn{7}{|l|}{$\begin{array}{l}\text { Ratio between the abdominal volume/the } \\
\text { volume of the hernia sac }\end{array}$} \\
\hline \multicolumn{7}{|l|}{ Primary versus incisional hernias } \\
\hline $\begin{array}{l}\text { Recurrent hernias (number of previous } \\
\text { repairs) }\end{array}$ & $x$ & $\times$ & $\times$ & $\times$ & $\times$ & $\times$ \\
\hline Previous mesh implantation & & & & & $x$ & \\
\hline $\begin{array}{l}\text { Indication for the operation causing the } \\
\text { incisional hernia }\end{array}$ & & & & & $\times$ & \\
\hline Type and localisation of the incision & & & & & $x$ & \\
\hline Symptoms of the hernia & & $\times$ & & & & \\
\hline Reducibility of the hernia & & $x$ & $x$ & & $x$ & \\
\hline Localisation of the hernia & $\times$ & $x$ & $x$ & $\times$ & $x$ & $\times$ \\
\hline $\begin{array}{l}\text { The anatomy of the patient in the } \\
\text { subcostal area: sternocostal angle }\end{array}$ & & & & & & $x$ \\
\hline Other risk factors for hernia recurrence & & & & & & $x$ \\
\hline
\end{tabular}

simple and practical to use. In Table 1 the potential variables are listed, as well as their use in previously proposed classifications. It is impossible to take all these variables into account for a practical classification, so a decision on inclusion or exclusion of various parameters was made.

\section{Classification of primary abdominal wall hernias}

For the primary abdominal wall hernias, there was agreement on the use of localisation and size as the two variables to use.

\section{Localisation of the hernia}

Two midline (epigastric and umbilical) and two lateral hernias (Spighelian and lumbar) are identifiable entities with distinct localisations.

\section{Size of the hernia}

Primary abdominal wall hernias are usually more or less round or oval shaped. Therefore, the size can be described with one measurement. Width and length will be more or less comparable most of the time. We agreed to use the "diameter" of the primary abdominal wall hernia as the second variable. Cutoff values of 2 and $4 \mathrm{~cm}$ were chosen to describe three subgroups according to size: small, medium and large.

\section{Taxomony}

For the primary abdominal wall hernias, the choice was made for nominative description: epigastric, umbilical, small, medium and large.

Classification table

In Table 2 the grid format for classification of primary abdominal wall hernias is proposed.

\section{Classification of incisional abdominal wall hernias}

\section{Definition of incisional hernia}

It was decided to use the definition proposed by Korenkov et al. [4]: "Any abdominal wall gap with or without a bulge in the area of a postoperative scar perceptible or palpable by clinical examination or imaging". 
Table 2 European Hernia Society classification for primary abdominal wall hernias

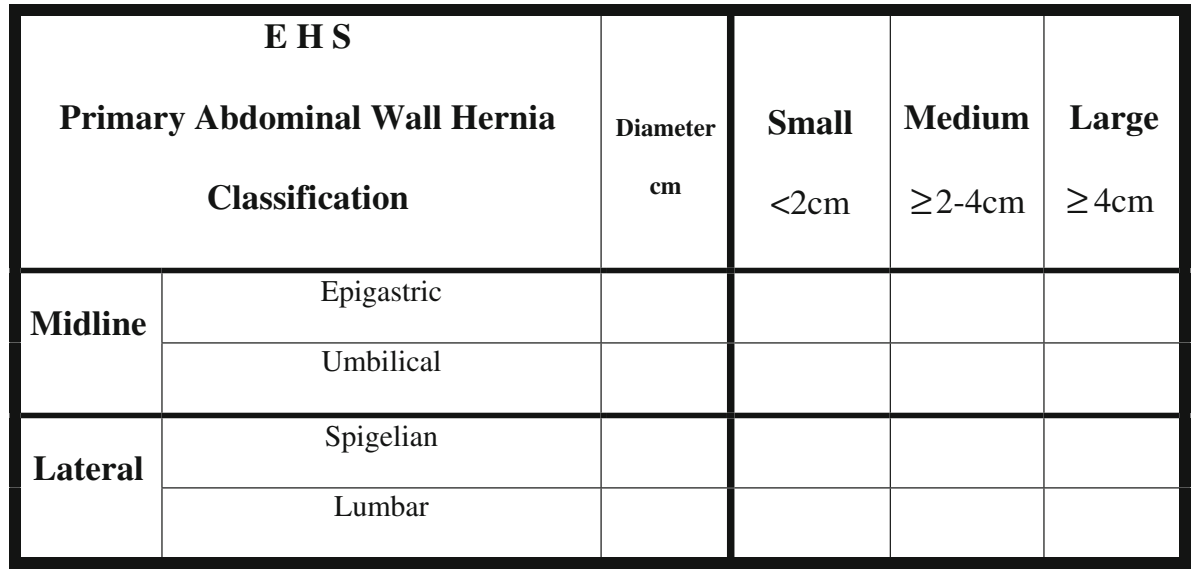

Choice of variables used to classify

The task of developing a good classification for incisional hernias is much more difficult than for groin hernias or for primary abdominal wall hernias because of their great diversity. On the other hand, because of this diversity a classification is highly desirable in this group of hernias. The question remains as to whether a simple classification can cover the complexities of the great diversity of incisional hernias and their different variables.

There was a consensus that the localisation of the hernia on the abdominal wall and the size of the hernia defect are essential for classifying. There was less agreement on the inclusion of the number of previous hernia repairs as a variable for classifying. Including more variables (Table 1) in the classification will make it more complex and less practical. Other variables and risk factors will be part of the above-mentioned registry, but for the present, will not be part of a simple classification.

Localisation of the hernia

The abdomen was divided into a medial or midline zone and a lateral zone.

\section{Medial or midline hernias}

The borders of the midline area are defined as:

(1) cranial: the xyphoid

(2) caudal: the pubic bone

(3) lateral: the lateral margin of the rectal sheath

Thus, all incisional hernias between the lateral margins of both rectus muscle sheaths are classified as midline hernias.

The Chevrel classification uses three midline zones [3]. Our group agreed that hernias close to bony structures have

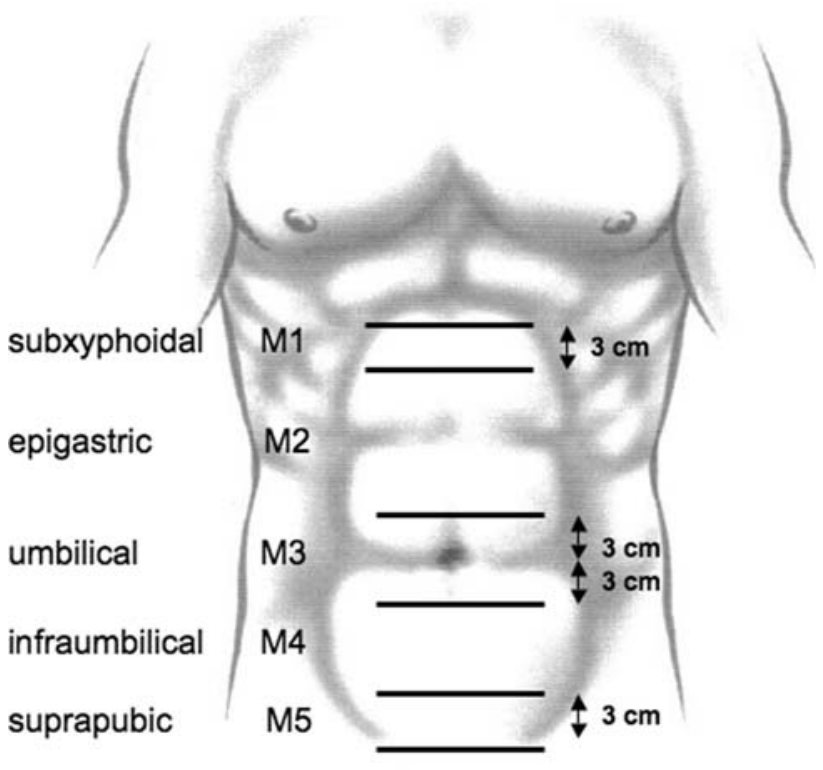

Fig. 1 To classify midline incisional hernias between the two lateral margins of the rectus muscle sheaths, five zones were defined

separate subgroups. They pose specific therapeutic approaches and have an increased recurrence risk. An easily memorable classification from M1 to M5 going from the xiphoid to pubic bone was proposed (Fig. 1). Therefore, we define $5 \mathrm{M}$ zones:

(1) M1: subxiphoidal (from the xiphoid till $3 \mathrm{~cm}$ caudally)

(2) M2: epigastric (from $3 \mathrm{~cm}$ below the xiphoid till $3 \mathrm{~cm}$ above the umbilicus)

(3) M3: umbilical (from $3 \mathrm{~cm}$ above till $3 \mathrm{~cm}$ below the umbilicus)

(4) M4: infraumbilical (from $3 \mathrm{~cm}$ below the umbilicus till $3 \mathrm{~cm}$ above the pubis)

(5) M5: suprapubic (from pubic bone till $3 \mathrm{~cm}$ cranially). 
Several questions arose from this classification:

(1) How should hernias extending over more than one $\mathrm{M}$ zone be classified? No consensus was reached on this. One proposal was to allocate hernias to the M zone that is generally considered as the more difficult or more representative for the hernia. They are, in order of importance: first subxyphoidal (M1) and suprapubic (M5), then umbilical (M3) and finally epigastric (M2) and infraumbilical (M4). This would avoid making further subgroups (e.g. M1-2/M1-2-3/M2-3-4). So a hernia extending from M1 over M2 to M3 (thus from subxyphoidal to the umbilicus) would be classified as M1 (thus as a subxiphoidal hernia). A hernia extending from M2 over M3 to M4 (thus from epigastric to infraumbilical) would be classified as M3 (thus as an umbilical hernia). No consensus was reached on this. It was decided to mark every zone in which the hernia was located when using the grid for incisional hernias.

(2) How should incisional hernias with multiple defects be classified? Different hernia defects caused by one incision will be considered as one hernia. If the different defects were caused by two different incisions, they should be considered two different hernias.

\section{Lateral hernias}

The borders of the lateral area are defined as (Fig. 2).

(1) cranial: the costal margin

(2) caudal: the inguinal region

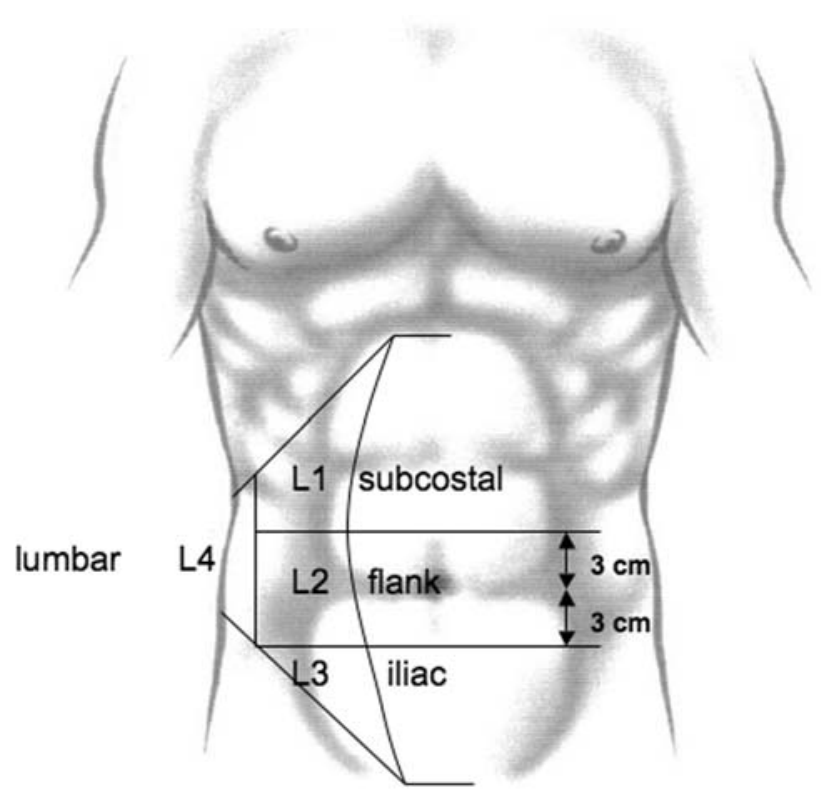

Fig. 2 To classify lateral incisional hernias, four zones lateral of the rectus muscle sheaths were defined
(3) medially: the lateral margin of the rectal sheath

(4) laterally: the lumbar region.

Thus, four L zones on each side are defined as:

(1) L1: subcostal (between the costal margin and a horizontal line $3 \mathrm{~cm}$ above the umbilicus)

(2) L2: flank (lateral to the rectal sheath in the area $3 \mathrm{~cm}$ above and below the umbilicus)

(3) L3: iliac (between a horizontal line $3 \mathrm{~cm}$ below the umbilicus and the inguinal region)

(4) L4: lumbar (latero-dorsal of the anterior axillary line)

\section{Taxomony}

Once subgroups had been defined, it was important to give them a name. Some of the experts were in favour of using simple coded notations similar to the Chevrel classification: M1, M2, M3,... L1, L2 ... W1, W2,... Others preferred a descriptive name: umbilical, supraumbilical, subcostal,... The advantage of a nominative description over a coded description is that it is more self-explanatory and comprehensible. No real consensus was reached over this topic, and a combination of coded and nominative descriptions is proposed.

Much discussion took place concerning the best word to describe the area on the lateral side of the abdomen below the subcostal region and above the iliac region. It was agreed that the word "transverse" as used in the Chevrel classification was not satisfactory. Finally, it was agreed to call this area the "flank".

\section{Size of the hernia}

In contrast to primary abdominal wall hernias, incisional hernias come in many different sizes and shapes. So the size of an incisional hernia is not easily captured in only one variable or measurement. For classification in the twodimensional grid format, it is essential to bring the variable "size of the hernia defect" in one quantitative or semiquantitative measure. Chevrel solved this problem by choosing the width of the hernia defect as the one parameter to classify, stating that the width is the most important measurement of size to determine the difficulty of succesfully repairing the hernia [3].

There was a consensus that the width of the hernia defect alone was insufficient to describe the hernia defect size adequately. We agreed that width and length should be used. This means that for a "grid format" both width and length have to be combined in one measurement.

The width of the hernia defect was defined as the greatest horizontal distance in $\mathrm{cm}$ between the lateral margins of the hernia defect on both sides. In case of 

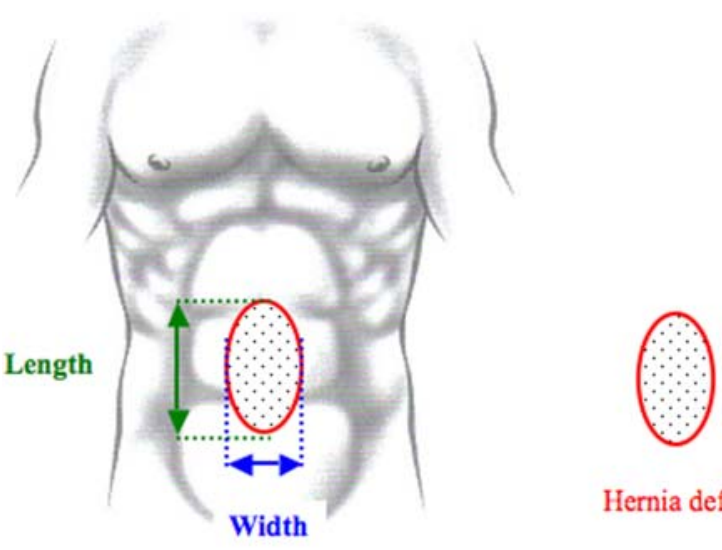

Hernia defect
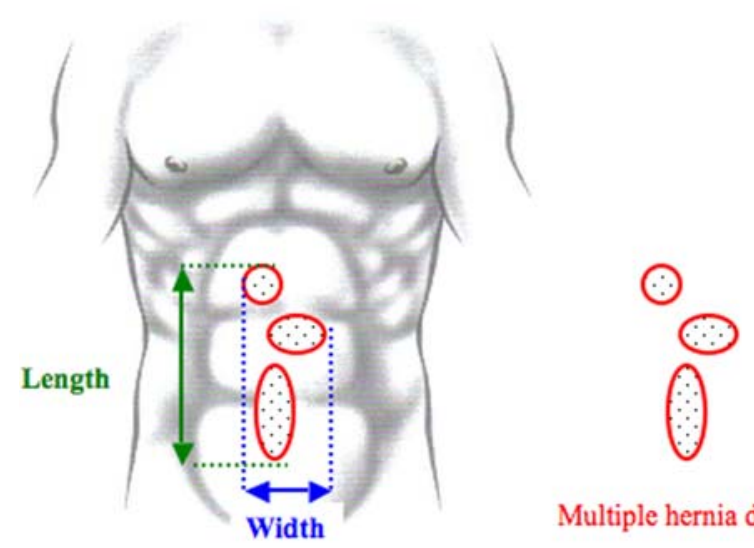

Multiple hernia defects

Fig. 3 Definition of the width and the length of incisional hernias for single hernia defects and multiple hernia defects

multiple hernia defects, the width is measured between the most laterally located margins of the most lateral defect on that side (Fig. 3).

The length of the hernia defect was defined as the greatest vertical distance in $\mathrm{cm}$ between the most cranial and the most caudal margin of the hernia defect. In case of multiple hernia defects from one incision, the length is between the cranial margin of the most cranial defect and the caudal margin of the most caudal defect (Fig. 3).

Hernia defect surface can be measured by combining width and length in a formula for an oval, thus trying to make an estimation of the real surface in $\mathrm{cm}^{2}$. This option was not withheld, because many incisional hernias are not oval shaped, and many hernias have multiple defects, making the correct estimation of hernia defect size difficult.

Because no consensus was reached on the variable "size of the hernia defect", it was not possible to make a "grid format" for an EHS classification for incisional abdominal wall hernias. Instead, the grid could be made for the localisation variable with space to note width and length correctly in $\mathrm{cm}$. A semi-quantitative division, taking only the width as measurement for the size, was accepted to be included in the classification table. To avoid confusion with primary abdominal wall hernias (small, medium and large), a coded taxonomy was chosen (W1 $<4 \mathrm{~cm}$; W2 $\geq$ $4-10 \mathrm{~cm} ; \mathrm{W} 3 \geq 10 \mathrm{~cm}$ ) instead of a nominative one.

Previous hernia repairs

Several participants in the meeting considered that if an incisional hernia is a recurrence after previous repair of a hernia-either incisional or primary-then this variable should be included in the classification. The number of previous hernia repairs was not considered of enough importance to include in the table. A simple yes or no answer was chosen.

Classification table

In Table 3 the format for classification of incisional abdominal wall hernias is proposed.

Table 3 European Hernia Society classification for incisional abdominal wall hernias

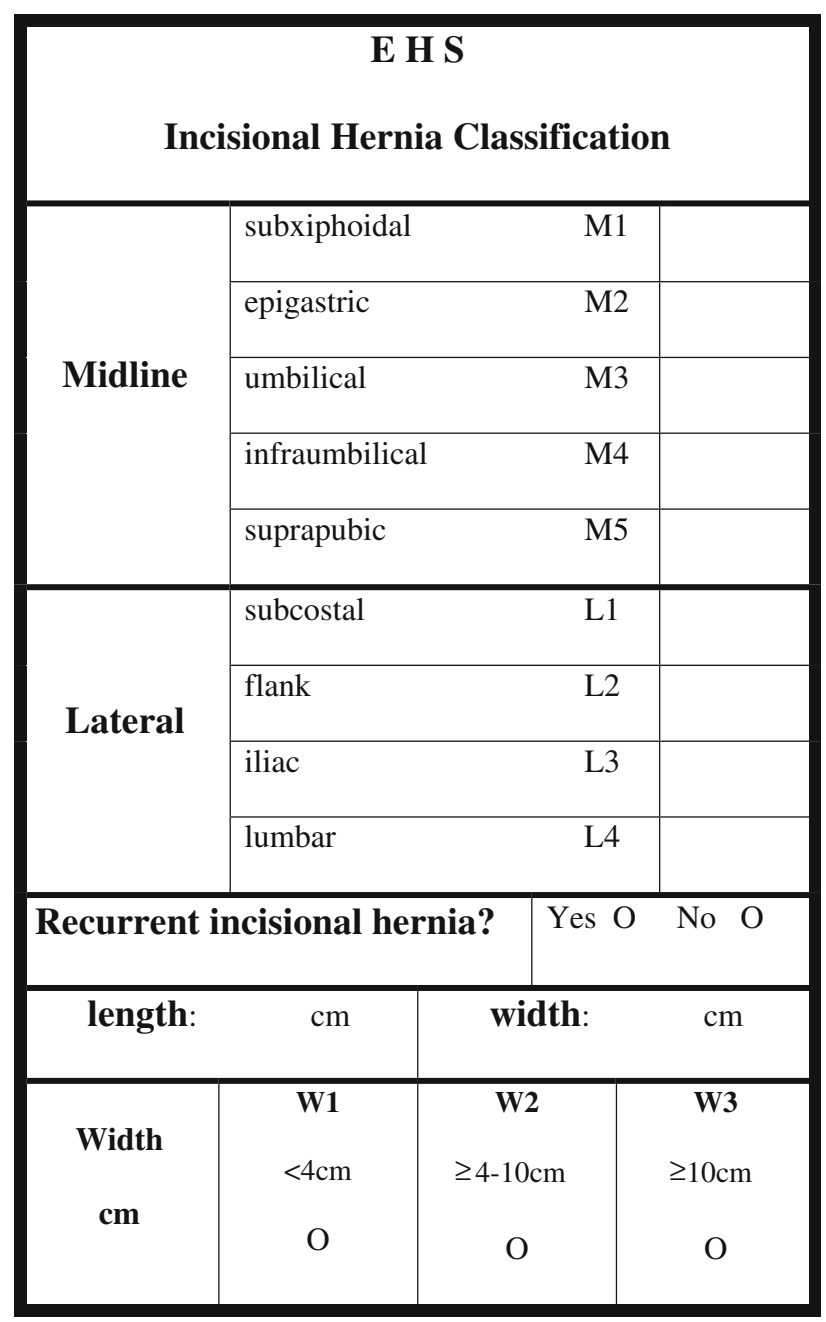




\section{Conclusion}

The goal of the consensus meeting, i.e. to make a definitive EHS classification of incisional hernias in a grid format, as has been done for inguinal hernias, was not realised. However, a classification for primary abdominal wall hernias and a division of subgroups of incisional abdominal wall hernias, concerning the localisation of the hernia, was formulated. Because no consensus was reached on a single size variable in incisional hernias, a simple classification grid was not possible.

Nevertheless, the participants in this meeting believe that, besides a more "scientific" registry (including risk factors, treatment and outcome data), a simple classification is urgently needed. This classification may provide enough information to establish incisional hernia registries and may be used to compare studies on treatment and outcome of incisional hernia repair. It has shortcomings, because of the large diversity and heterogeneity of incisional hernias, but it is a mandatory condition to improve the quality of reporting results in the field of incisional hernia surgery.

Therefore, we must use the momentum created by this first consensus meeting on classification of primary and incisional abdominal wall hernias. The current proposal should be tested and validated in our surgical practices. This will provide a basis for a new consensus meeting to try to define subgroups based on the size of the hernia defect.

Open Access This article is distributed under the terms of the Creative Commons Attribution Noncommercial License which permits any noncommercial use, distribution, and reproduction in any medium, provided the original author(s) and source are credited.

\section{References}

1. Inauguration speech of the new president of the European Hernia Society, Andrew Kingsnorth, at the 29th International Congress of the European Hernia Society, 6 May 2007, Athens, Greece

2. Schumpelick V (2000) Narbenhernie. In: Schumpelick V (ed) Hernien. Thieme, Stuttgart, pp 266-269

3. Chevrel JP, Rath AM (2000) Classification of incisional hernias of the abdominal wall. Hernia 4:7-11

4. Korenkov M, Paul A, Sauerland S, Neugebauer E, Arndt M, Chevrel JP, Corcione F, Fingerhut A, Flament JB, Kux M, Matzinger A, Myrvold HE, Rath AM, Simmermacher RK (2001) Classification and surgical treatment of incisional hernia. Results of an experts' meeting. Langenbecks Arch Surg 386:65-73

5. Dietz UA, Hamelmann W, Winkler MS, Debus ES, Malafaia O, Czeczko NG, Thiede A, Kuhfu $\beta$ I (2007) J Plast Reconstr Aesthet Surg 60(4):383-388

6. Ammaturo C, Bassi G (2005) The ratio between anterior abdominal wall surface/wall defect surface: a new parameter to classify abdominal incisional hernias. Hernia 9:316-321

7. Miserez M et al (2007) The European hernia society groin hernia classification: simple and easy to remember. Hernia 11(2):113-116 